\title{
PARAMETERIZATIONS AND PROBABILITY DISTRIBUTIONS OF ORIENTATIONS
}

\author{
H. SCHAEBEN
}

Laboratory of Metallurgy of Polycrystalline Materials (LM2P), University of Metz, Ile du Saulcy, 57045 Metz, France;

and BASYS Software GmbH, Peterstr. 2-4, 5100 Aachen, W. Germany

(Received May 8, 1990)

\begin{abstract}
General normal distributions of orientations (proper rotations) with respect to different parameterizations are mathematically rigorously and notationally concisely represented. This includes their explicit formulations as well as their characterizations in terms of maximum entropy and their explict one-one correspondence.
\end{abstract}

KEY WORDS Orientation statistics, von Mises-Fisher distribution, orthogonal group $\left(\mathrm{SO}_{3}\right)^{+}$, Rodrigues parameters, directional statistics, Bingham distribution.

\section{INTRODUCTION}

In quantitative texture analysis (QTA) an orientation $g$ of a crystal grain has conventionally been defined as a proper rotation in terms of Eulerian angles $(\alpha, \beta, \gamma)$. Adapting matrix notation the space $G$ of orientations $g$ is identified with the orthogonal group $\left(\mathrm{SO}_{3}\right)^{+}$of $(3 \times 3)$ orthogonal matrices $R$ with determinant equal to +1 .

\section{RODRIGUES PARAMETERS}

However, besides the Eulerian angles there are other different parameterizations of rotations and hence orientations (Becker and Panchanadeeswaran, 1989; Morawiec and Pospiech, 1989). The Rodrigues parameters $\left(r_{1}, r_{2}, r_{3}, r_{4}\right)$ of a rotation provide the most convenient representation of an orientation for statistical purposes (Altmann, 1986; Moran, 1975). They may be thought of as components of a quaternion, or as components of a unit vector $\vec{r} \in S^{4}$ as $\sum_{i=1}^{4} r_{i}^{2}=1$. Since $\vec{r} \in S^{4}$ and $-\vec{r} \in S^{4}$ define the same rotation (orientation), an orientation $g$ is uniquely determined by a point of the upper (lower) fourdimensional unit hemisphere $S_{+}^{4}$, or equivalently by a point of the projective hyperplane $H^{4}$ constituted by identifying $\vec{r}$ and $-\vec{r}, \vec{r} \in S_{+}^{4}$.

\section{BINGHAM DISTRIBUTION OF ORIENTATIONS}

Thus, statistics of orientation data require probability distributions on $S_{+}^{4}$. A mathematical model is provided by the Bingham distribution (Bingham, 1964; 
1974). Let $\vec{r} \in S_{+}^{4} ; \vec{r}$ is said to be distributed as $B_{4}(L, A)$ if $\vec{r}$ has the Bingham distribution

$$
\left(c_{B}(L)\right)^{-1} \exp \left\{\operatorname{tr}\left(L A \vec{r} \vec{r}^{t} A^{t}\right)\right\}[d s]
$$

where [ds] represents the Lebesgue invariant area element on $S_{+}^{4}, A$ is a $(4 \times 4)$ orthogonal matrix, $L$ is a $(4 \times 4)$ diagonal matrix with entries $l_{1}, \ldots, l_{4}$, and $c_{B}(L)$ is a normalizing constant depending on $L$ only. Different sets of values of $L$ in Eq. (1) give the uniform distribution, a girdle or a bipolar distribution. It should be noted that $B_{4}\left(L+\alpha I_{4}, A\right)$ and $B_{4}(L, A)$ are indistinguishable for any $\alpha \in \mathbb{R}^{1}$, i.e. the shape parameters $l_{i}, i=1, \ldots, 4$, are determined only up to an additive constant. Uniqueness can be imposed by some convention, e.g. $l_{4}=0$.

\subsection{Characterization of the Bingham Distribution}

There are several useful characterizations of the $p$-variate Bingham distribution, two of which will be repeated here. Obviously, it is closely related to the $p$-variate normal distribution. Actually, $B_{4}(L, A)$ is the conditional distribution given $\|\vec{r}\|=1$ when $\vec{r} \in \mathbb{R}^{4}$ has a four-variate normal distribution $N_{4}(0, \Sigma)$ with zero mean and covariance matrix $\Sigma=-\frac{1}{2}\left(A L A^{t}\right)^{-1}$ (Bingham, 1974).

Another characterization is given in terms of maximum entropy. For given "moment of inertia" $E\left(\overrightarrow{\vec{r}}^{t}\right)$ the Bingham distribution is the distribution on $S_{+}^{4}$ with maximum entropy (Mardia, 1974).

Here, the maximum entropy principle provided a constructive criterion for determining a probability distribution on the base of partial knowledge in the form of summary statistics or equivalently constraints related to population parameters such as $E\left(\vec{r}^{t}\right)$ (Mardia, 1975).

\section{VON MISES-FISHER DISTRIBUTION OF ORIENTATIONS}

Due to the one-one correspondence between $\left(\mathrm{SO}_{3}\right)^{+}$and $\mathrm{S}_{+}^{4}$, respectively $\mathrm{H}^{4}$, a probability distribution of unit vectors $\vec{r} \in S_{+}^{4}$ determines another probability distribution of $(3 \times 3)$ orthogonal matrices $R \in\left(\mathrm{SO}_{3}\right)^{+}$, and vice versa.

A mathematical model of a probability distribution of orientations represented by $(3 \times 3)$ orthogonal matrices $R \in\left(\mathrm{SO}_{3}\right)^{+}$is provided by the von Mises-Fisher matrix distribution (Khatri and Mardia, 1977). Let $R \in\left(\mathrm{SO}_{3}\right)^{+}$; then $R$ is said to be distributed as $M_{3}(F)$ if $R$ has the von Mises-Fisher matrix density

$$
\left(c_{M}(F)\right)^{-1} \exp \left\{\operatorname{tr}\left(F R^{t}\right)\right\}[d R]
$$

where $[d R]$ represents the uniform distribution on $\left(\mathrm{SO}_{3}\right)^{+}, \mathrm{F}$ is a $(3 \times 3)$ parameter matrix, and $c_{M}(F)$ is a normalizing constant depending on $F$. $F$ may be represented as

$$
F=K M
$$

where $K$ is the elliptical, and $M$ the polar component of $F$, with $M \in\left(\mathrm{SO}_{3}\right)^{+}$ (Khatri and Mardia, 1977). 


\subsection{Characterization of the von Mises-Fisher Distribution}

A characterization of $M_{3}(F)$ in terms of trivariate normal distributions may be given as follows. If the three rows of $\mathrm{R} \in\left(\mathrm{SO}_{3}\right)^{+}$are independent observations from a trivariate normal distribution with covariance matrix $K^{-1}$ and mean vectors the corresponding rows of $M$, where $M M^{t}=I_{3}$ since $M \in\left(S_{3}\right)^{+}$, then the distribution of $R$ given $R R^{t}=I_{3}$ is $M_{3}(F)$ with $F=K M$.

Also, $\mathrm{M}_{3}(\mathrm{~F})$ is the probability distribution on $\left(\mathrm{SO}_{3}\right)^{+}$with maximum entropy for given expectation $E(R)$ (Mardia, 1974).

\section{CORRESPONDENCE OF BINGHAM AND VON MISES-FISHER DISTRIBUTION}

Employing the one-one correspondence between $\left(\mathrm{SO}_{3}\right)^{+}$and $\mathrm{S}_{+}^{4}$ the following theorem has been deduced.

$R \in\left(\mathrm{SO}_{3}\right)^{+}$has a von Mises-Fisher matrix distribution $\mathrm{M}_{3}(\mathrm{~F})$ if and only if $\vec{r} \in S_{+}^{4}$ has a Bingham distribution $B_{4}(L, A)$ where their parameters are related as

$$
\operatorname{tr}\left(F R^{t}\right)=\vec{r}^{t} A L A^{t} \vec{r}
$$

where $R \in\left(S_{3}\right)^{+}$and $\vec{r} \in S_{+}^{4}$ are equivalent representations of the same orientation $g$ (Prentice, 1986).

\section{APPLICATIONS IN QUANTIATIVE TEXTURE ANALYSIS}

Choosing $F=\kappa I_{3}, K \in \mathbb{R}^{1}$, and adapting the notation $\tilde{g}=I_{3} R^{t}$ where $\tilde{g}$ may be interpreted as the distance of the rotation $R$ from the identical rotation $I_{3}$, we readily recognize

$$
N_{1}(\kappa) \exp \{\kappa \operatorname{tr}(\tilde{g})\}=N_{2}(\kappa) \exp (\kappa \cos \tilde{\omega}), \quad 0 \leq \tilde{\omega} \leq \pi
$$

which has been labeled standard Gaussian distribution by Matthies (1980), and recently discussed in some length (Matthies et al., 1988), as the special case of the von Mises-Fisher orientation distribution when it is central. In fact, the right hand side of Eq. (5) is nothing else but the classic von Mises-Fisher distribution of directions (Mardia, 1974).

The general von Mises-Fisher orientation distribution explicitly given in Eqs. $(2,3)$ may be used to generate mathematical model orientation density functions (odf) which are not central. Non-central model odfs may prove useful in studies of preferred orientation in non-metals.

Also, the explicit formulation of the general Bingham and the von MisesFisher orientation distribution respectively is the essential prerequisite of proper statistical inference.

\section{CONCLUSION}

The rigorous treatise of normal distributions of orientations with respect to different parameterizations should aid in a better understanding of the mathematical background of quantitative texture analysis and its practical problems. 
The inversion problem of QTA and its ambiguity will be discussed in terms of multivariate statistics (as opposed to functional analysis) elsewhere.

\section{Acknowledgement}

The manuscript has been prepared while its author held an invited professorship at the University of Metz, France. Special thanks for this invitation as well as for stimulating discussions are due to Prof. C. Esling, M. Humbert, and F. Wagner.

\section{References}

1. Altmann, S. L. (1986) Rotations, Quaternions, and the Double Group, Oxford University Press, Oxford.

2. Becker, R. and Panchanadeeswaran, S. (1989) Textures and Microstructures, 10, 167-194.

3. Bingham, C. (1964) Ph.D. Thesis, Yale University.

4. Bingham, C. (1974) Ann. Statist., 2, 1201-1225.

5. Khatri, C. G. and Mardia, K. V. (1977) J. Roy. Statist. Soc., ser. B, 39, 95-106.

6. Mardia, K. V. (1974) in Statistical Distributions in Scientific Work, vol. 3, edited by Patil, G. P., Kotz, S., and Ord, J. K. (D. Reidel Publishing Company, Dordrecht), 365-385.

7. Mardia, K. V. (1975) J. Roy. Statist. Soc., ser. B, 37, 349-393.

8. Matthies, S. (1980) phys. stat. sol. (b), 101, K111-115.

9. Matthies, S., Muller, J., and Vinel, G. W. (1988) Textures and Microstructures, 10, 77-96.

10. Moran, P. A. P. (1975) in Perspectives in Probability and Statistics, edited by Gani, J. (Applied Probability Trust), 295-301.

11. Morawiec, A. and Pospiech, J. (1989) Textures and Microstructures, 10, 167-194.

12. Prentice, M. J. (1986) J. Roy. Statist. Soc., ser. B, 48, 214-222. 